\title{
MINIMAL ENERGY POINT SYSTEMS ON THE UNIT CIRCLE AND THE REAL LINE
}

\author{
MARCELL GAÁL*, BÉLA NAGY ${ }^{\dagger}$, ZSUZSANNA NAGY-CSIHA ${ }^{\ddagger}$, AND SZILÁRD \\ GY. RÉVÉSZ*
}

\begin{abstract}
.
In this paper, we investigate discrete logarithmic energy problems in the unit circle. We study the equilibrium configuration of $n$ electrons and $n-1$ pairs of external protons of charge $+1 / 2$. It is shown that all the critical points of the discrete logarithmic energy are global minima, and they are the solutions of certain equations involving Blaschke products. As a nontrivial application, we refine a recent result of Simanek, namely, we prove that any configuration of $n$ electrons in the unit circle is in stable equilibrium (that is, they are not just critical points but are of minimal energy) with respect to an external field generated by $n-1$ pairs of protons.
\end{abstract}

Key words. Blaschke product, electrostatic equilibrium, potential theory, external fields

AMS subject classifications. 31C20, 30J10, 78A30

1. Introduction and preliminaries. The motivation of this work comes from certain equilibrium questions which, in turn, have roots in rational orthogonal systems. Exploring the connection between critical points of orthogonal polynomials and equilibrium points goes back to Stieltjes. For more on this connection, see, e.g., [9], [10] and the references therein.

Rational orthogonal systems are widely used on the area of signal processing, and also on the field of system and control theory. These systems consist of rational functions with poles located outside the closed unit disk. A wide class of rational orthogonal systems is the so-called Malmquist-Takenaka system from which one can recover the usual trigonometric system, the Laguerre system and the Kautz system as well. In earlier works, in analogy with the discrete Fourier transform, a discretized version of the Malmquist-Takenaka system was introduced.

In signal processing and system identification (e.g. mechanical systems related to control theory) the rational orthogonal bases and Malmquist-Takenaka systems (e.g. discrete Laguerre and Kautz systems) are more efficient than the trigonometric system in the determination of the transfer functions. There are lots of results in this field, see e.g. [3] and the references therein, or [13] and [7].

In connection with potential theory, it was studied (e.g. in [14]) whether the discretization nodes satisfy certain equilibrium conditions, namely, whether they arise from critical points of a logarithmic potential energy. Such discretizations appear naturally, see e.g. [1] by Bultheel et al or [5] by Golinskii. The question whether the critical points are minima was proposed by Pap and Schipp [14, 15]. In this paper, we follow this line of research. After this introduction and statements of results, we study on the unit circle a quite general logarithmic energy which is determined by a signed measure, and prove that after inverse Cayley transform the transformed energy on the real line differs only in an additive constant. Next using a recent result of Semmler and Wegert [16] we give an affirmative answer to the question posed by

\footnotetext{
*Alfréd Rényi Institute of Mathematics, Budapest, Hungary (gaal.marcell@renyi.hu, revesz.szilard@renyi.hu).

${ }^{\dagger}$ MTA-SZTE Analysis and Stochastics Research Group, University of Szeged, Szeged, Hungary (nbela@math.u-szeged.hu).

${ }^{\ddagger}$ Institute of Mathematics and Informatics, University of Pécs, (ncszsu@gamma.ttk.pte.hu) and Department of Numerical Analysis, Eötvös Loránd University, Budapest, Hungary .
} 
Pap and Schipp concerning the critical points. Finally, as an application, we present a refinement of a result of Simanek [18].

First let us start with some notation and essential background material. We use the standard notations $\mathbb{D}:=\{z \in \mathbb{C}:|z|<1\}, \partial \mathbb{D}:=\{z \in \mathbb{C}:|z|=1\}$, $\mathbb{D}^{*}:=\{z \in \mathbb{C}:|z|>1\}, \mathbb{T}:=\mathbb{R} / 2 \pi \mathbb{Z}$ and $\zeta^{*}:=1 / \bar{\zeta}(\zeta \neq 0)$. We also use Blaschke products, defined for $a_{1}, \ldots, a_{n} \in \mathbb{D}$ and $\chi,|\chi|=1$ as

$$
B(z):=\chi \prod_{k=1}^{n} \frac{z-a_{k}}{1-\overline{a_{k}} z} .
$$

In particular, when the leading coefficient $\chi=1, B(z)$ is called monic Blaschke product.

We assume $B^{\prime}(0) \neq 0$. In this case the well-known Walsh' Blaschke theorem (see for instance [17], p. 377) says that $B^{\prime}(z)=0$ has $2 n-2$ (not necessarily different) solutions, where $n-1$ of them (counted with multiplicites) are in the unit disk, and if $\zeta \in \mathbb{D} \backslash\{0\}$ satisfies $B^{\prime}(\zeta)=0$, then $\zeta^{*}:=1 / \bar{\zeta}$ is also a critical point, $B^{\prime}\left(\zeta^{*}\right)=0$, with the same multiplicity as $\zeta$. It also follows that then $\left.B^{\prime}\right|_{\partial \mathbb{D}} \neq 0$.

Next, we investigate the structure of solutions of the equation

$$
B\left(e^{i t}\right)=e^{i \delta},
$$

where $B($.$) is a Blaschke product. It is standard to see that \Im \log B\left(e^{i t}\right)$ can be defined continuously and it is strictly increasing on $[0,2 \pi]$ from

$$
\alpha:=\Im \log B(1)=\arg B(1), \quad \alpha \in[-\pi, \pi)
$$

to $\alpha+2 n \pi$, see, e.g. [17], pp. 373-374. Therefore (1.2) has $n$ different solutions in $t \in[0,2 \pi)$ for any $\delta \in \mathbb{R}$. Hence it is logical to consider $n$-tuples of different solutions as solution vectors for (1.2).

Now, we are to reduce different types of symmetries among the solution vectors step-by-step. For given $\delta \in \mathbb{R}$, consider

$$
\left\{\left(\tau_{1}, \ldots, \tau_{n}\right) \in \mathbb{R}^{n}: B\left(e^{i \tau_{j}}\right)=e^{i \delta}, j=1, \ldots, n\right\} .
$$

We can restrict our attention to the reduced set $\tau_{1} \leq \tau_{2} \leq \ldots \leq \tau_{n} \leq \tau_{1}+2 \pi$ without loss of generality, for picking any $\tau_{1}$ we can normalize mod $2 \pi$ and then order the remaining $\tau_{j}$. Actually, since the $\tau_{j}$ are different, all such solutions of (1.2) belong to the open set

$$
A:=\left\{\left(\tau_{1}, \tau_{2}, \ldots, \tau_{n}\right) \in \mathbb{R}^{n}: \tau_{1}<\tau_{2}<\ldots<\tau_{n}<\tau_{1}+2 \pi\right\} .
$$

It is a standard step (see [17] loc. cit.) that one can define the functions $\delta \mapsto \tau_{j}(\delta)$ such that they are continuously differentiable, strictly increasing, and $\tau_{1}(\delta)<\ldots<$ $\tau_{n}(\delta)<\tau_{1}(\delta)+2 \pi$ for all $\delta \in \mathbb{R}$, while $B\left(\exp \left(i \tau_{j}(\delta)\right)\right)=\exp (i \delta) j=1, \ldots, n$. As $B\left(e^{i 0}\right)=e^{i \alpha}$, we have $0 \in\left\{\tau_{1}(\alpha), \tau_{2}(\alpha), \ldots, \tau_{n}(\alpha)\right\}$. By relabelling again, if necessary, we may assume that

$$
\tau_{1}(\alpha)=0
$$

Hence $T(\delta):=\left(\tau_{1}(\delta), \ldots, \tau_{n}(\delta)\right)$ can be viewed as a smooth arc lying in $A \subset \mathbb{R}^{n}$. Moreover, the graph $S_{\mathbb{R}}:=\{T(\delta): \delta \in \mathbb{R}\}$ contains all the solutions of (1.2) from $A$, that is, if $\mathbf{t}:=\left(t_{1}, \ldots, t_{n}\right) \in A$ and $\lambda \in \mathbb{R}$ are such that $B\left(\exp \left(i t_{j}\right)\right)=\exp (i \lambda), j=$ 
$1, \ldots, n$ hold, then there exists $\delta \in \mathbb{R}$ such that $\mathbf{t}=T(\delta)$. Furthermore, $\exp \left(i \tau_{j}(\delta+\right.$ $2 n \pi))=\exp \left(i \tau_{j}(\delta)\right)$ for $j=1,2, \ldots, n, \delta \in \mathbb{R}$. We introduce the set

$$
S_{0}:=S_{\mathbb{R}} \cap[0,2 \pi)^{n}=\{T(\delta): \delta \in[\alpha, \alpha+2 \pi)\}
$$

where we used (1.5). We call the set

$$
S:=\{T(\delta): \delta \in[\alpha, \alpha+2 n \pi)\}
$$

the solution curve. Note that

$$
\begin{aligned}
& S=S_{\mathbb{R}} \cap Q, \text { where } \\
& Q:=[0,2 \pi) \times\left[\tau_{2}(\alpha), \tau_{2}(\alpha)+2 \pi\right) \times \ldots \times\left[\tau_{n}(\alpha), \tau_{n}(\alpha)+2 \pi\right)
\end{aligned}
$$

where we also used $(1.5)$, so $\left[\tau_{1}(\alpha), \tau_{1}(\alpha)+2 \pi\right)=[0,2 \pi)$. Geometrically, $S$ can be obtained from $S_{0}$ with reflections and translations, while $S_{\mathbb{R}}$ can be obtained from $S$ with translations only. Another useful property of $S$ is that for each $\beta \in[0,2 \pi)$ there is exactly one $\delta \in[\alpha, \alpha+2 n \pi)$ such that $\tau_{1}(\delta)=\beta$.
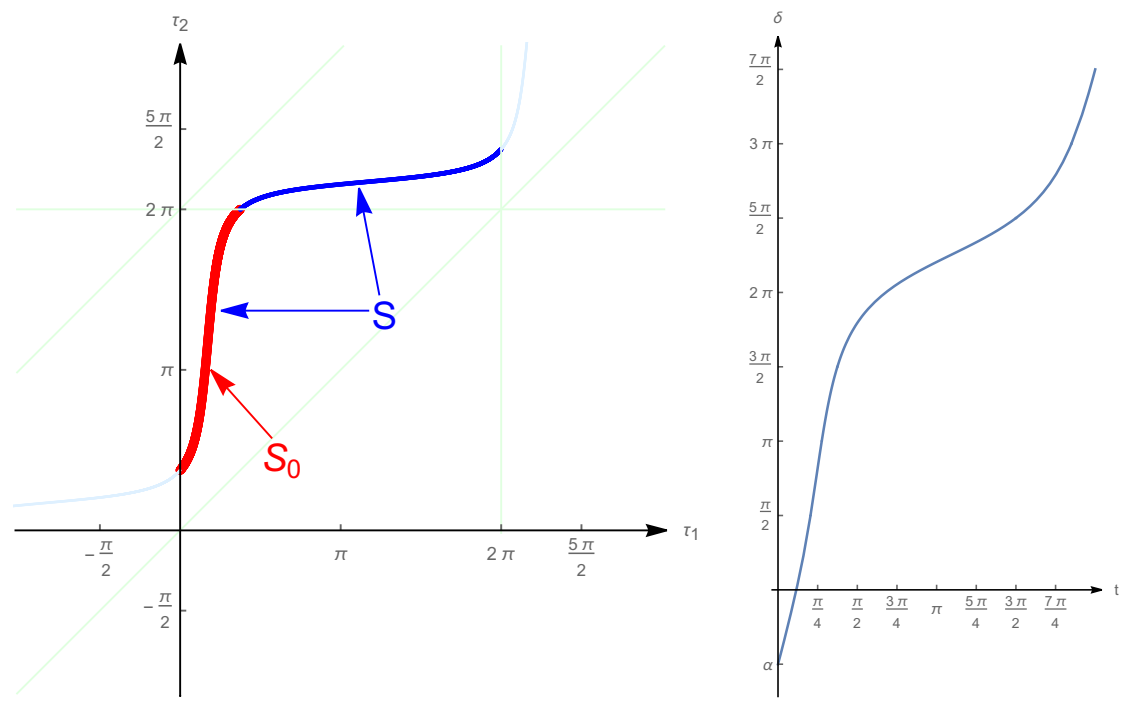

FIGURE 1. Left: solution curve $S$ of the monic Blaschke product with zeros at $1 / 2$ and $(1+i) / 2$, $0 \leq \tau_{1}<\tau_{2}<2 \pi, B\left(e^{i \tau_{1}}\right)=B\left(e^{i \tau_{2}}\right)=e^{i \delta}, \alpha \leq \delta \leq \alpha+2 \pi$, where $\alpha=-\pi / 2$ now. Right: argument of the same monic Blaschke product, $\delta=\arg \bar{B}\left(e^{i t}\right)$.

These are depicted on the left half of Figure 1 where $S_{0}$ is the thick arc and it is continued above with another arc. These two arcs together form $S$ and describe the motions of $\tau_{1}, \tau_{2}$ together as $\exp (i \delta)$ goes around the unit circle twice ( $\delta$ grows from $\alpha$ to $\alpha+4 \pi)$. Extending these two arcs with the very thin arcs, we obtain $S_{\mathbb{R}}$, the full solution curve.

Now we recall the question raised by Pap and Schipp in [15]. Consider the pairs of protons, each of charge $+1 / 2$, at $\zeta_{1}, \zeta_{1}^{*}, \ldots, \zeta_{n-1}, \zeta_{n-1}^{*}$ as the critical points of a (monic) Blaschke product of degree $n$, and the (doubled) discrete energy of electrons restricted to the unit circle

$$
W\left(w_{1}, \ldots, w_{n}\right):=\sum_{k=1}^{n-1} \sum_{j=1}^{n} \log \left|\left(w_{j}-\zeta_{k}\right)\left(w_{j}-\zeta_{k}^{*}\right)\right|-2 \sum_{1 \leq j<k \leq n} \log \left|w_{j}-w_{k}\right|
$$


where $\left|w_{1}\right|=1, \ldots,\left|w_{n}\right|=1$. The set $S_{\mathbb{R}}$ connected to the same monic Blaschke product yields critical configurations of electrons for each fixed $\delta$ (which corresponds to fixing one of the electrons), according to e.g. [15]. In other words, for $a_{1}, \ldots, a_{n} \in \mathbb{D}$, using the monic Blaschke product with zeros at $a_{1}, \ldots, a_{n}$ one can construct pairs of protons as solutions of $B^{\prime}(z)=0$, and, for any given $\delta \in[0,2 \pi)$, the corresponding configuration of electrons as all solutions of $B(z)=e^{i \delta}$. Then according to the result of Pap and Schipp, Theorem 4 from [15], these configurations of electrons are critical points of $W$. The question posed on p. 476 of [15] is then: Are these critical points (local) minima of the restricted energy function $\widetilde{W}$ where $\widetilde{W}\left(\tau_{1}, \ldots, \tau_{n}\right):=$ $W\left(e^{i \tau_{1}}, \ldots, e^{i \tau_{n}}\right), \tau_{1} \ldots, \tau_{n} \in \mathbb{R} ?$

We give a positive answer to this question in general. Note that two special cases were solved in [15] with different methods. Our answer is the following. There are no other critical points on the unit circle (where the tangential gradient vanishes). Moreover, all the points on the set $S_{\mathbb{R}}$ are global minimum points of the restricted energy function $\widetilde{W}$.

TheOREM 1.1. Let $a_{1}, \ldots, a_{n} \in \mathbb{D}$ and $B(z)$ be the monic Blaschke product (1.1) with zeros at $a_{1}, \ldots, a_{n}$. Assuming $B^{\prime}(0) \neq 0$, list up the critical points of $B$ as $\zeta_{1}, \ldots, \zeta_{n-1} \in \mathbb{D} \backslash\{0\}$ and $\zeta_{1}^{*}, \ldots, \zeta_{n-1}^{*} \in \mathbb{D}^{*}$.

Then the tangential gradient of $W$ vanishes on the points corresponding to the set $A \cap Q$ defined in (1.4) exactly on the set $S$.

More precisely, on $A \cap Q$, it holds that $\nabla \widetilde{W}\left(\tau_{1}, \ldots, \tau_{n}\right)=0$ if and only if $\left(\tau_{1}, \ldots, \tau_{n}\right)=T(\delta)$ for some $\delta \in[\alpha, \alpha+2 n \pi)$.

Furthermore, all points of $S_{\mathbb{R}}$ are global minimum points of $\widetilde{W}$.

Let us recall here a recent result of Simanek [18, Theorem 2.1]. Briefly, he established that for any configuration of electrons on the unit circle, there is an external field (collection of protons) such that the electrons are in electrostatic equilibrium (that is, the gradient of the energy is zero). We are going to refine this result by determining the number of pairs of protons and their locations using the solution curve defined in (1.7).

For the following we need some more results on Blaschke products. Namely for given $z_{1}, z_{2}, \ldots, z_{n} \in \mathbb{C},\left|z_{j}\right|=1, z_{j} \neq z_{k}(j \neq k)$, we need to find a Blaschke product $B($.$) of degree m$, such that

$$
B\left(z_{j}\right)=\chi \prod_{k=1}^{m} \frac{z_{j}-a_{k}}{1-\overline{a_{k}} z_{j}}=1, \quad j=1,2, \ldots, n .
$$

The first result of this kind was established by Cantor and Phelps in [2] (for some $m$ ) and the stronger form with degree $m \leq n-1$ was given by Jones and Ruscheweyh in [11], see also a paper by Hjelle [8]. By using the results of Jones and Ruscheweyh, Hjelle showed that there is a Blaschke product $B(z)$ of degree $m=n$ such that (1.9) holds, see [8], p. 44. We will use this particular Blaschke product $B(z)=$ $B\left(z_{1}, z_{2}, \ldots, z_{n} ; z\right)$ corresponding to $z_{1}, z_{2}, \ldots, z_{n}$. Note that Hjelle's Blaschke product is not unique, since there is an extra iterpolation condition. Observe that the extra interpolation condition can be chosen so that $B^{\prime}(0) \neq 0$ is satisfied.

TheOrem 1.2. For distinct $z_{1}, \ldots, z_{n} \in \partial \mathbb{D}$ fix a Blaschke product $B(z)$ so that (1.9) holds with $m=n$ and $B^{\prime}(0) \neq 0$. Denote the critical points of $B(z)$ in the unit disk by $\zeta_{1}, \zeta_{2}, \ldots, \zeta_{n-1}$.

Then the (doubled) energy function $W\left(w_{1}, \ldots, w_{n}\right)$, constructed by means of these points $\zeta_{1}, \zeta_{2}, \ldots, \zeta_{n-1}$ according to $(1.8)$, has critical point at $\left(w_{1}, \ldots, w_{n}\right)=\left(z_{1}, \ldots, z_{n}\right)$ 
(even regarded as a point of $\left.\mathbb{C}^{n}\right)$.

Moreover, on $(\partial \mathbb{D})^{n},\left.W\right|_{(\partial \mathbb{D})^{n}}$ has global minimum at $\left(z_{1}, \ldots, z_{n}\right)$.

2. Some basic propositions. Recall that it was given in (1.8) the discrete energy of an electron configuration $w_{1}, \ldots, w_{n} \in \mathbb{C}$ (with charges -1 ) in presence of an external field generated by pairs of fixed protons $\zeta_{1}, \zeta_{1}^{*}, \zeta_{2}, \zeta_{2}^{*}, \ldots, \zeta_{n-1}, \zeta_{n-1}^{*}$ (with charges $+1 / 2$ each), where $\zeta_{1}, \ldots, \zeta_{n-1} \in \mathbb{D}$. Note that actually $W$ is the double of the physical energy of the system (see also [12], p. 22 where they use this form of discrete energy). We will see later on why it is more convenient to use this "doubled energy".

Sometimes the following exceptional set will be excluded:

$$
\begin{aligned}
E:=\left\{\left(w_{1}, \ldots, w_{n}, \zeta_{1}, \ldots, \zeta_{n-1}\right) \in \mathbb{C}^{n} \times \mathbb{D}^{n-1}:\right. & \\
\zeta_{j}=0 \text { for some } j \text { or } w_{j}= & w_{k} \text { for some } j \neq k \\
& \text { or } \left.\zeta_{j}=w_{k} \text { or } \zeta_{j}^{*}=w_{k} \text { for some } j, k\right\} .
\end{aligned}
$$

This is a closed set with empty interior. Geometrically, this set covers the cases when some of the protons are at the origin, some of the electrons are at the same position or a proton and an electron are at the same position. Let us remark also that $W=$ $W\left(w_{1}, \ldots, w_{n}\right)$ is locally the real part of a holomorphic function when $\zeta_{1}, \ldots, \zeta_{n-1}$ are fixed and $W$ is considered on $\left(w_{1}, \ldots, w_{n}\right) \in \mathbb{C}^{n}$ such that $\left(w_{1}, \ldots, w_{n}, \zeta_{1}, \ldots, \zeta_{n-1}\right) \notin$ E.

This energy can be generalized substantially. Let $\mu$ be a signed measure on $\mathbb{C}$. We define the (doubled) energy in this case as

$$
\begin{gathered}
W_{\mu, 1}:=2 \sum_{k=1}^{n} \int_{\mathbb{C}} \log \left|w_{k}-\zeta\right| d \mu(\zeta), \quad W_{\mu, 2}:=\sum_{\substack{l \neq k \\
1 \leq l, k \leq n}} \log \left|w_{l}-w_{k}\right|, \text { and } \\
W_{\mu}\left(w_{1}, \ldots, w_{n}\right):=W_{\mu, 1}-W_{\mu, 2} .
\end{gathered}
$$

Note that in (1.8) we sum over all $l<k$ pairs and there is an extra factor 2. In (2.2), the sum is over all $l \neq k$ pairs. Later this second, symmetric expression will be more convenient.

Here, it may happen that $W_{\mu, 1}$ or $W_{\mu, 2}$ becomes infinity, so we again introduce the exceptional set as follows:

$$
\begin{aligned}
E_{\mu}:=\left\{\left(w_{1}, \ldots, w_{n}\right) \in \mathbb{C}^{n}:\right. & w_{j}=w_{k} \text { for some } j \neq k \\
& \text { or } \left.\int_{\mathbb{C}}|\log | w_{j}-\zeta|| d|\mu|(\zeta)=+\infty \text { for some } j\right\} .
\end{aligned}
$$

Note that finiteness of this latter integral is equivalent to the finiteness of the potentials of $\mu_{+}$and $\mu_{-}$at $w_{j}$ where $\mu_{+}, \mu_{-}$are the positive and negative parts of $\mu$ respectively. Observe that if $\left(w_{1}, \ldots, w_{n}\right) \notin E_{\mu}$, then $W_{\mu, 1}$ and $W_{\mu, 2}$ are finite, and so is $W_{\mu}$.

An important tool in our investigations is the Cayley transform and its inverse. Basically, it is just a transformation between a half-plane and the unit disk, though there is no widely accepted, standard form of it. We use the following form, which we call inverse Cayley transform

$$
C(z)=C_{\theta}(z):=i \frac{1+z e^{-i \theta}}{1-z e^{-i \theta}}
$$


where $\theta \in \mathbb{R}$ will be specified later. It is standard to verify that $C(z)$ maps the unit disk onto the upper half-plane, $C_{\theta}\left(e^{i \theta}\right)=\infty$, and $C($. $)$ maps bijectively the unit circle (excluding $\left.e^{i \theta}\right)$ to the real axis. Furthermore, $C_{\theta}\left(e^{i t}\right)$ is continuous and strictly increasing from $t=\theta$ to $t=\theta+2 \pi, C_{\theta}\left(e^{i t}\right) \rightarrow-\infty$ as $t \rightarrow \theta+0, C_{\theta}\left(e^{i t}\right) \rightarrow+\infty$ as $t \rightarrow \theta+2 \pi-0$. It is easy to see that $C\left(z^{*}\right)=\overline{C(z)}$ and $C^{\prime}(z) \neq 0$ (if $\left.z \neq e^{i \theta}\right)$. Later we will use the Cayley transform too:

$$
C_{\theta}^{-1}(u)=e^{i \theta} \frac{u-i}{u+i} .
$$

Mapping the electrons and protons by $C_{\theta}$, we define $t_{j}$ with $t_{j}=C_{\theta}\left(w_{j}\right)$. We also write $\xi_{j}:=C_{\theta}\left(\zeta_{j}\right)$ and accordingly, $\overline{\xi_{j}}=C_{\theta}\left(\zeta_{j}^{*}\right)$ and investigate the following new discrete energy:

$$
V\left(t_{1}, \ldots, t_{n}\right):=\sum_{k=1}^{n-1} \sum_{j=1}^{n} \log \left|\left(t_{j}-\xi_{k}\right)\left(t_{j}-\bar{\xi}_{k}\right)\right|-2 \sum_{1 \leq j<k \leq n} \log \left|t_{j}-t_{k}\right| .
$$

We also define the (doubled) discrete energy on the real line when the external field is determined by a signed measure $\nu$ :

$$
\begin{gathered}
V_{\nu, 1}:=2 \sum_{k=1}^{n} \int_{\mathbb{C}} \log \left|t_{k}-\xi\right| d \nu(\xi), \quad V_{\nu, 2}:=\sum_{\substack{l \neq k \\
1 \leq l, k \leq n}} \log \left|t_{l}-t_{k}\right| \text { and } \\
V_{\nu}\left(t_{1}, \ldots, t_{n}\right):=V_{\nu, 1}-V_{\nu, 2} .
\end{gathered}
$$

We introduce again the exceptional set corresponding to $\nu$ as follows:

$$
\begin{aligned}
& E_{\nu}:=\left\{\left(t_{1}, \ldots, t_{n}\right) \in \mathbb{C}^{n}: t_{j}=t_{k} \text { for some } j \neq k\right. \\
& \left.\qquad \text { or } \int_{\mathbb{C}}|\log | t_{j}-\xi|| d|\nu|(\xi)=+\infty \text { for some } j\right\} .
\end{aligned}
$$

The next result gives a somewhat surprising connection how the inverse Cayley transform carries over energy. Actually, there is a cancellation in the background which makes it work.

Proposition 2.1. Fix $\theta \in \mathbb{R}$ and let $\mu$ be a signed measure on $\mathbb{C}$ with compact support such that $\mu(\{0\})=0, \mu(\mathbb{C})=n-1$. Write $\nu:=\mu \circ C_{\theta}^{-1}$, that is, $\nu(B)=$ $\mu\left(C_{\theta}^{-1}(B)\right)$ for every Borel set $B$.

Assume that $w_{1}, \ldots, w_{n} \in \mathbb{C}$ and $\left(w_{1}, \ldots, w_{n}\right) \notin E_{\mu}$ and

$$
\int_{\mathbb{C}} \log \left|\zeta-e^{i \theta}\right| d \mu(\zeta) \text { is finite. }
$$

Then with $t_{1}, \ldots, t_{n} \in \mathbb{C}$ where $t_{j}=C_{\theta}\left(w_{j}\right)$, we know that $\left(t_{1}, \ldots, t_{n}\right) \notin E_{\nu}$, $W_{\mu}\left(w_{1}, \ldots, w_{n}\right)$ and $V_{\nu}\left(t_{1}, \ldots, t_{n}\right)$ are finite and we can write

$$
W_{\mu}\left(w_{1}, \ldots, w_{n}\right)=V_{\nu}\left(t_{1}, \ldots, t_{n}\right)+c
$$

where $c$ is a finite constant, namely

$$
c=n(n-1) \log (2)-2 n \int_{\mathbb{C}} \log |\xi+i| d \nu(\xi) .
$$


Proof. It is straightforward to verify that $\left(t_{1}, t_{2}, \ldots, t_{n}\right) \notin E_{\nu}$. Furthermore,

$$
\begin{array}{r}
\int_{\mathbb{C}} \log |\xi+i| d \nu(\xi)=\int_{\mathbb{C}} \log \left|C_{\theta}(\zeta)+i\right| d \mu(\zeta)=\int_{\mathbb{C}} \log \left|i\left(1+\frac{1+\zeta e^{-i \theta}}{1-\zeta e^{i \theta}}\right)\right| d \mu(\zeta) \\
=\int_{\mathbb{C}} \log (2)-\log \left|\zeta-e^{i \theta}\right| d \mu(\zeta),
\end{array}
$$

so (2.6) is equivalent to

$$
\int_{\mathbb{C}} \log |\xi+i| d \nu(\xi) \text { is finite. }
$$

Note that this entails the finiteness of $c$ defined in (2.8).

With the notation of the Proposition,

$$
\begin{aligned}
& W_{\mu}\left(w_{1}, \ldots, w_{n}\right)-V_{\nu}\left(t_{1}, \ldots, t_{n}\right)=2 \sum_{k=1}^{n} \int_{\mathbb{C}} \log \left|w_{k}-\zeta\right| d \mu(\zeta) \\
& -\sum_{\substack{j \neq k \\
1 \leq j, k \leq n}} \log \left|w_{j}-w_{k}\right|-2 \sum_{k=1}^{n} \int_{\mathbb{C}} \log \left|t_{k}-\xi\right| d \nu(\xi)+\sum_{\substack{j \neq k \\
1 \leq j, k \leq n}} \log \left|t_{j}-t_{k}\right|
\end{aligned}
$$

where we investigate the difference of the integrals and difference of the sums separately. So we write

$$
\begin{gathered}
\int_{\mathbb{C}} \log \left|w_{k}-\zeta\right| d \mu(\zeta)-\int_{\mathbb{C}} \log \left|t_{k}-\xi\right| d \nu(\xi) \\
=\int_{\mathbb{C}} \log \left|C_{\theta}^{-1}\left(t_{k}\right)-C_{\theta}^{-1}(\xi)\right| d \nu(\xi)-\int_{\mathbb{C}} \log \left|t_{k}-\xi\right| d \nu(\xi) \\
=\int_{\mathbb{C}} \log \left|e^{i \theta}\left(\frac{t_{k}-i}{t_{k}+i}-\frac{\xi-i}{\xi+i}\right)\right|-\log \left|t_{k}-\xi\right| d \nu(\xi) \\
=\int_{\mathbb{C}} \log (2)+\log \left|\frac{1}{\left(t_{k}+i\right)(\xi+i)}\right| d \nu(\xi) \\
=\int_{\mathbb{C}}-\log |\xi+i| d \nu(\xi)+\left(\log (2)-\log \left|t_{k}+i\right|\right) \nu(\mathbb{C}),
\end{gathered}
$$

where this last integral exists, by assumption (2.9). Similarly,

$$
\begin{array}{r}
\log \left|t_{j}-t_{k}\right|-\log \left|w_{j}-w_{k}\right|=\log \left|t_{j}-t_{k}\right|-\log \left|C_{\theta}^{-1}\left(t_{j}\right)-C_{\theta}^{-1}\left(t_{k}\right)\right| \\
=\log \left|t_{j}-t_{k}\right|-\log \left|e^{i \theta}\left(\frac{t_{j}-i}{t_{j}+i}\right)-e^{i \theta}\left(\frac{t_{k}-i}{t_{k}+i}\right)\right| \\
=-\log (2)+\log \left|t_{j}+i\right|+\log \left|t_{k}+i\right| .
\end{array}
$$


Substituting into (2.10), we get

$$
\begin{aligned}
& W_{\mu}\left(w_{1}, \ldots, w_{n}\right)- V_{\nu}\left(t_{1}, \ldots, t_{n}\right) \\
&=2 \sum_{k=1}^{n}\left(\int_{\mathbb{C}}-\log |\xi+i| d \nu(\xi)+\left(\log (2)-\log \left|t_{k}+i\right|\right) \nu(\mathbb{C})\right) \quad+\sum_{\substack{j \neq k \\
1 \leq j, k \leq n}}\left(-\log (2)+\log \left|t_{j}+i\right|+\log \left|t_{k}+i\right|\right) \\
&=-2 \nu(\mathbb{C}) \sum_{k=1}^{n} \log \left|t_{k}+i\right|+2 n \nu(\mathbb{C}) \log (2)-2 n \int_{\mathbb{C}} \log |\xi+i| d \nu(\xi) \\
& \quad-n(n-1) \log (2)+2(n-1) \sum_{k=1}^{n} \log \left|t_{k}+i\right| \\
&=n(n-1) \log (2)-2 n \int_{\mathbb{C}} \log |\xi+i| d \nu(\xi),
\end{aligned}
$$

where we used that $\nu(\mathbb{C})=n-1$.

REMARK 2.2. Since $\mu$ has compact support, $\operatorname{supp} \nu$ is disjoint from $-i$, moreover, their distance is positive. Hence the logarithm in the integral in (2.8) is bounded from below. It is not necessarily bounded from above, but we assume (2.9) directly. Instead of supposing (2.9), we may suppose that $\mu$ and $\theta$ (from Cayley transform) are such that $\operatorname{supp} \mu$ and $e^{i \theta}$ are of positive distances from each other. This would ensure that $\operatorname{supp} \nu$ remains bounded entailing that the logarithm in the integral in (2.9) is bounded from above. In other words, if $\operatorname{supp} \mu$ is compact and $e^{i \theta} \notin \operatorname{supp} \mu$, then (2.9) holds.

We note that this Proposition 2.1 extends the result of Theorem 6 in Pap, Schipp [15] that we allow arbitrary signed external fields in place of discrete protons located symmetrically with respect to the unit circle.

Proposition 2.3. We maintain the assumptions and notations of Proposition 2.1. Let $\ell \in\{1, \ldots, n\}$ and let $w_{j}, j \neq \ell$ be fixed.

Assume that

$$
e^{i \theta} \notin \operatorname{supp} \mu
$$

and assume further that replacing $w_{\ell}$ by $e^{i \theta}$, we have

$$
\left(w_{1}, \ldots, e^{i \theta}, \ldots, w_{n}\right) \notin E_{\mu} .
$$

If $w_{\ell} \rightarrow e^{i \theta}$, then $\left|t_{\ell}\right|=\left|C_{\theta}\left(w_{\ell}\right)\right| \rightarrow \infty$ and we get that

$$
W_{\mu}\left(w_{1}, \ldots, w_{\ell-1}, e^{i \theta}, w_{\ell+1}, \ldots, w_{n}\right)=V_{\nu}\left(t_{1}, \ldots, t_{\ell-1}, \infty, t_{\ell+1}, \ldots, t_{n}\right)+c
$$

where $c$ is the constant defined in (2.8) and

$$
\begin{aligned}
V_{\nu}\left(t_{1}, \ldots, t_{\ell-1}, \infty, t_{\ell+1}, \ldots, t_{n}\right):=V_{\nu}\left(t_{1}, \ldots, t_{\ell-1}, t_{\ell+1}, \ldots, t_{n}\right) \\
=2 \sum_{\substack{j=1 \\
j \neq \ell}}^{n} \int_{\mathbb{C}} \log \left|t_{j}-\xi\right| d \nu(\xi)-\sum_{\substack{1 \leq j, k \leq n \\
j \neq \ell, k \neq \ell, j \neq k}} \log \left|t_{j}-t_{k}\right| .
\end{aligned}
$$


Proof. First, we discuss why the integrals appearing here are finite. By slightly abusing the notation, $W_{\mu}\left(w_{\ell}\right):=W_{\mu}\left(w_{1}, \ldots, w_{\ell}, \ldots, w_{n}\right)$ is finite at $w_{\ell}=e^{i \theta}$, because of (2.12). Assumption (2.11) implies that there is a neighborhood $U$ of $e^{i \theta}$ such that its closure $U^{-}$is disjoint from $\operatorname{supp} \mu, U^{-} \cap \operatorname{supp} \mu=\emptyset$. Therefore $W_{\mu}(w)$ is also finite when $w \in U$, moreover $W_{\mu}($.$) is continuous there. Similarly, we use V_{\nu}(t):=$ $V_{\nu}\left(t_{1}, \ldots, t_{\ell-1}, t, t_{\ell+1}, \ldots, t_{n}\right)$ (abusing the notation again). Obviously, $C_{\theta}(U)$ is an unbounded open set on the extended complex plane $\mathbb{C}_{\infty}$ and is a neighborhood of infinity. By Proposition 2.1, $V_{\nu}(t)$ is defined on $C_{\theta}(U) \backslash\{\infty\}$, has finite value and is continuous there. Moreover, $V_{\nu}(t)$ has finite limit as $t \rightarrow \infty$. By (2.12) and (2.11), $\left(w_{1}, \ldots, w_{\ell-1}, w, w_{\ell+1}, \ldots, w_{n}\right) \notin E_{\mu}$ for $w \in U$. Hence $\left(t_{1}, \ldots, t_{\ell-1}, t, t_{\ell+1}, \ldots, t_{n}\right) \notin$ $E_{\nu}$ for $t \in C_{\theta}(U) \backslash\{\infty\}$. This also implies that $\int_{\mathbb{C}} \log \left|t_{j}-\xi\right| d \nu(\xi)$ is finite, $j=1, \ldots, n$, $j \neq \ell$, which are the integrals appearing on the right of (2.14).

Regarding $V_{\nu}$, we write

$$
\begin{aligned}
& \lim _{t_{\ell} \rightarrow \infty} V_{\nu}\left(t_{\ell}\right)=\lim _{t_{\ell} \rightarrow \infty}\left(2 \sum_{j=1}^{n} \int_{\mathbb{C}} \log \left|t_{j}-\xi\right| d \nu(\xi)-\sum_{\substack{1 \leq j, k \leq n \\
j \neq k}} \log \left|t_{j}-t_{k}\right|\right) \\
& =2 \sum_{\substack{j=1 \\
j \neq \ell}}^{n} \int_{\mathbb{C}} \log \left|t_{j}-\xi\right| d \nu(\xi)-\sum_{\substack{1 \leq j, k \leq n \\
j \neq \ell, k \neq \ell}} \log \left|t_{j}-t_{k}\right| \\
& +\lim _{t_{\ell} \rightarrow \infty}\left(2 \int_{\mathbb{C}} \log \left|t_{\ell}-\xi\right| d \nu(\xi)-\sum_{\substack{1 \leq j, k \leq n \\
k \neq j, k=\ell \text { or } j=\ell}} \log \left|t_{j}-t_{k}\right|\right) \\
& =V\left(t_{1}, \ldots, t_{\ell-1}, t_{\ell+1}, \ldots, t_{n}\right),
\end{aligned}
$$

where in the last step we used the following calculation.

$$
\begin{aligned}
\lim _{t_{\ell} \rightarrow \infty}\left(2 \int_{\mathbb{C}} \log \left|t_{\ell}-\xi\right| d \nu(\xi)-\sum_{\substack{1 \leq j, k \leq n \\
k \neq j, k=\ell}} \operatorname{or} j=\ell\right. \\
\quad=\lim _{t_{\ell} \rightarrow \infty} 2 \int_{\mathbb{C}} \log \left|t_{\ell}\right|+\log \left|1-\frac{\xi}{t_{\ell}}\right| d \nu(\xi)-2 \sum_{\substack{1 \leq j \leq n \\
j \neq \ell}}\left(\log \left|t_{\ell}\right|+\log \left|1-\frac{t_{j}}{t_{\ell}}\right|\right)
\end{aligned}
$$

where $\int_{\mathbb{C}} \log \left|t_{\ell}\right| d \nu(\xi)=(n-1) \log \left|t_{\ell}\right|$ so the first term in the integral and in the sum cancel each other, by $\nu(\mathbb{C})=n-1$. Regarding the second term in the sum, it tends to zero. The second term in the integral also tends to zero, because the support of $\nu$ is compact, hence $\log \left|1+\xi / t_{\ell}\right|$ tends to 0 uniformly.

Using this calculation, (2.7) from Proposition 2.1 and the properties of $W_{\mu}$ and $C_{\theta}$ we get that

$$
\begin{aligned}
& W_{\mu}\left(e^{i \theta}\right)=\lim _{w_{\ell} \rightarrow e^{i \theta}} W_{\mu}\left(w_{\ell}\right) \\
&=\lim _{t_{\ell} \rightarrow \infty}\left(V_{\nu}\left(t_{\ell}\right)+c\right)=V_{\nu}\left(t_{1}, \ldots, t_{\ell-1}, t_{\ell+1}, \ldots, t_{n}\right)+c .
\end{aligned}
$$


Based on the above proposition, it is justified to extend the definition of $V_{\nu}$ by continuity as $V_{\nu}\left(t_{1}, \ldots, t_{\ell-1}, \infty, t_{\ell+1}, \ldots, t_{n}\right):=V_{\nu}\left(t_{1}, \ldots, t_{\ell-1}, t_{\ell+1}, \ldots, t_{n}\right)$ in case $t_{\ell}$ becomes $\pm \infty$.

Now we are going to relate the critical points of $W_{\mu}$ and $V_{\nu}$ when the configurations of the electrons are restricted to the unit circle (or to the real line).

When the electrons are restricted to the unit circle, that is,

$$
\left|w_{j}\right|=1, \quad j=1, \ldots, n
$$

we are going to introduce the tangential gradient as follows. In this case, in addition to supposing that $\mu$ has compact support, we assume that $\operatorname{supp} \mu$ is disjoint from the unit circle.

We write

$$
w_{j}=e^{i \tau_{j}}, \quad j=1, \ldots, n, \quad \widetilde{W}_{\mu}\left(\tau_{1}, \ldots, \tau_{n}\right):=W_{\mu}\left(e^{i \tau_{1}}, \ldots, e^{i \tau_{n}}\right) .
$$

We call $\nabla \widetilde{W}_{\mu}$ the tangential gradient of $W_{\mu} . \nabla \widetilde{W}_{\mu}$ of $\widetilde{W_{\mu}}$ has special meaning with respect to the complex derivative of $W_{\mu}$ : it is the tangential component of $\nabla W_{\mu}$ with respect to the unit circle. Similar distinction also appears in [18], see the definitions of $\Gamma$-normal electrostatic equilibrium and total electrostatic equilibrium on p. 2255. This total electrostatic equilibrium appears in Theorem 2, [14] which will be used later.

Proposition 2.4. Let $\nu$ be a signed measure on $\mathbb{C}$ with compact support. Assume that $\operatorname{supp} \nu$ is disjoint from the real line and $\nu$ is symmetric with respect to the real line: $\nu(H)=\nu(\bar{H})$ where $H \subset\{\Im(u)>0\}$ is a Borel set and $\bar{H}=\{\bar{u}: u \in H\}$ denotes the complex conjugate.

Then for $u_{1}, \ldots, u_{n} \in \mathbb{R}$ we have for the $j$-th imaginary directional derivative (with direction $i \mathbf{e}_{j}:=i(0, \ldots, 0,1,0, \ldots, 0)$ ) that

$$
\begin{aligned}
\partial_{i \mathbf{e}_{j}} V_{\nu}\left(u_{1}, \ldots, u_{n}\right) & \\
& :=\lim _{v_{j} \rightarrow 0} \frac{V_{\nu}\left(u_{1}, \ldots, u_{j}+i v_{j}, \ldots, u_{n}\right)-V_{\nu}\left(u_{1}, \ldots, u_{n}\right)}{v_{j}}=0 .
\end{aligned}
$$

Roughly speaking, if the external field is symmetric, then the forces moving the electrons will keep the electrons on the real line (all coordinates of gradient are parallel with the real line).

Proposition 2.5. Let $\mu$ be a signed measure on $\mathbb{C}$ with compact support. Assume that $\operatorname{supp} \mu$ is disjoint from the unit circle and $\mu$ is symmetric with respect to the unit circle: $\mu(H)=\mu\left(H^{*}\right)$ where $H \subset\{|w|<1\}$ is a Borel set and $H^{*}=\{1 / \bar{w}: w \in H\}$ denotes the inversion of $H$.

Then for $\left|w_{1}\right|=\ldots=\left|w_{n}\right|=1$, we have for the $j$-th normal derivative (with direction $w_{j} \mathbf{e}_{j}$ ) that

$$
\begin{aligned}
\partial_{w_{j} \mathbf{e}_{j}} W_{\mu}\left(w_{1}, \ldots, w_{n}\right) & \\
& :=\lim _{\varepsilon \rightarrow 0} \frac{W_{\mu}\left(w_{1}, \ldots, w_{j}+\varepsilon w_{j}, \ldots, w_{n}\right)-W_{\mu}\left(w_{1}, \ldots, w_{n}\right)}{\varepsilon}=0 .
\end{aligned}
$$

Note that because $\mu$ has compact support and is symmetric with respect to the unit circle, we necessarily have that 0 is not in $\operatorname{supp} \mu$. 
Roughly speaking, Proposition 2.5 states that if the measure $\mu$ is symmetric with respect to the unit circle, then the gradient and the tangential gradient of $W_{\mu}$ are the same. In other words, $n$ electrons on the unit circle, allowed to move freely on the plane in the external field generated by $\mu$ will stay on the unit circle.

Proofs of Propositions 2.4 and 2.5. To see Proposition 2.4, we fix $u_{1}, \ldots, u_{j-1}$, $u_{j}, u_{j+1}, \ldots, u_{n} \in \mathbb{R}$, and use here $J($.$) for the conjugation: J(u)=\bar{u}$. Writing $V(u):=V_{\nu}\left(u_{1}, \ldots, u_{j-1}, u, u_{j+1}, \ldots, u_{n}\right)$ for general complex $u=u_{j}+i v_{j}$, and using that $\nu$ is symmetric to the real line, in other words, $\nu(H)=\nu(J(H))$ for Borel sets $H$, we find

$$
V\left(u_{1}, \ldots, u_{j-1}, u, u_{j+1}, \ldots, u_{n}\right)=V\left(u_{1}, \ldots, u_{j-1}, J(u), u_{j+1}, \ldots, u_{n}\right) .
$$

Therefore,

$$
\begin{aligned}
& \partial_{i \mathbf{e}_{j}} V\left(u_{1}, \ldots, u_{j-1}, u_{j}, u_{j+1}, \ldots, u_{n}\right) \\
& =\left.\frac{\partial V\left(u_{1}, \ldots, u_{j-1}, u_{j}+i v_{j}, u_{j+1}, \ldots, u_{n}\right)}{\partial v_{j}}\right|_{\left(u_{1}, \ldots, u_{j-1}, u_{j}, u_{j+1}, \ldots, u_{n}\right)} \\
& =\left.\frac{\partial V\left(u_{1}, \ldots, u_{j-1}, u_{j}-i v_{j}, u_{j+1}, \ldots, u_{n}\right)}{\partial v_{j}}\right|_{\left(u_{1}, \ldots, u_{j-1}, u_{j}, u_{j+1}, \ldots, u_{n}\right)} \\
& =\left.\frac{\partial V\left(u_{1}, \ldots, u_{j-1}, u_{j}+i v_{j}, u_{j+1}, \ldots, u_{n}\right)}{\partial\left(-v_{j}\right)}\right|_{\left(u_{1}, \ldots, u_{j-1}, u_{j}, u_{j+1}, \ldots, u_{n}\right)} \\
& =-\partial_{i \mathbf{e}_{j}} V\left(u_{1}, \ldots, u_{j-1}, u_{j}, u_{j+1}, \ldots, u_{n}\right)
\end{aligned}
$$

showing that Proposition 2.4 holds.

To see Proposition 2.5, we use that the inverse Cayley transform is a conformal mapping, hence it is locally orthogonal.

3. The case of finitely many pairs of protons. In this section, we specialize the propositions of the previous section. Most of the results here simply follow from those statements.

We consider the case when $\operatorname{supp} \mu$ is a finite set with $2 n-2$ elements, which are symmetric with respect to the unit circle and the support is disjoint from the unit circle and the origin:

$$
\begin{gathered}
\operatorname{supp} \mu=\left\{\zeta_{1}, \zeta_{2}, \ldots, \zeta_{n-1}, \zeta_{1}^{*}, \zeta_{2}^{*}, \ldots, \zeta_{n-1}^{*}\right\} \\
0<\left|\zeta_{j}\right|<1, \mu\left(\left\{\zeta_{j}\right\}\right)=\mu\left(\left\{\zeta_{j}^{*}\right\}\right)=1 / 2, \quad j=1,2, \ldots, n-1, \\
\zeta_{j} \neq \zeta_{k}, \quad j, k=1,2, \ldots, n-1, \quad j \neq k
\end{gathered}
$$

Recall that $\zeta^{*}=1 / \bar{\zeta}$.

The restriction $\zeta_{j} \neq 0$ is essential for the following reasons. Although $0^{*}=\infty$ may be introduced, definition of discrete energy $W$ cannot be meaningfully defined. Note that the usefulness of symmetrization of external fields lies in that the normal component of the field generated by the symmetrized proton configuration identically vanishes on the unit circle. However, when there is a proton at the origin, there is no complementing system of protons $\omega_{1}, \ldots, \omega_{m}$ (for no $m$ ) such that the total system $\left\{\zeta_{1}, \ldots, \zeta_{n}, \omega_{1}, \ldots, \omega_{m}\right\}$ would generate a field with identically vanishing normal component on the unit circle.

Furthermore, the protons at the origin contribute to the electrostatic field of all protons only with identically zero tangential component all over the unit circle. 
Therefore, studying equilibrium and energy minima on the circle, protons at the origin have no contribution, hence can be dropped from the configuration. However, then the total charge of the system will drop below -1 . There are results in this essentially different case, too, see e.g. [6] or [4], Theorem 4.1 but those necessarily involve assumptions on locations of electrons.

The below Proposition 3.1 follows directly from the more general Proposition 2.1. Roughly speaking, it expresses how the energy functions are mapped to one another via the inverse Cayley transform in this special case. We use here the exceptional set $E$ introduced in (2.1).

Proposition 3.1. Fix $\theta \in \mathbb{R}$ and let $\zeta_{j} \in \mathbb{D}, j=1, \ldots, n-1$. Consider the parameters $\zeta_{j}, \zeta_{j}^{*}$ as well as the parameters $\xi_{j}=C_{\theta}\left(\zeta_{j}\right), \overline{\xi_{j}}=C_{\theta}\left(\zeta_{j}^{*}\right)$.

Assume that $w_{1}, \ldots, w_{n} \in \mathbb{C}$ are such that $\left(w_{1}, \ldots, w_{n}, \zeta_{1}, \ldots, \zeta_{n-1}\right) \notin E$, and $w_{j} \neq e^{i \theta}(j=1, \ldots, n)$.

With $t_{1}, \ldots, t_{n} \in \mathbb{C}$ where $t_{j}=C_{\theta}\left(w_{j}\right)$, we can write

$$
W\left(w_{1}, \ldots, w_{n}\right)=V\left(t_{1}, \ldots, t_{n}\right)+c
$$

where $c$ is a constant,

$$
c=n(n-1) \log (2)-n \sum_{k=1}^{n-1} \log \left|\left(\xi_{k}+i\right)\left(\bar{\xi}_{k}+i\right)\right| .
$$

If $\left(w_{1}, \ldots, w_{n}, \zeta_{1}, \ldots, \zeta_{n-1}\right) \in E$, then $W, V$ or $c$ is infinite.

Next we formulate the following special case of Proposition 2.3.

Proposition 3.2. Let $\ell \in\{1, \ldots, n\}$ and let $w_{j}, j \neq \ell$ be fixed such that $w_{j} \neq e^{i \theta}$ for all $j \neq \ell$. If $w_{\ell}=e^{i \theta}$, then $t_{\ell}=C_{\theta}\left(w_{\ell}\right)=\infty$ and we get that

$$
W\left(w_{1}, \ldots, w_{\ell-1}, e^{i \theta}, w_{\ell+1}, \ldots, w_{n}\right)=V\left(t_{1}, \ldots, t_{\ell-1}, \infty, t_{\ell+1}, \ldots, t_{n}\right)+c
$$

where $c$ is defined in (3.2) and similarly to (2.14)

$$
\begin{aligned}
V\left(t_{1}, \ldots, t_{\ell-1}, \infty, t_{\ell+1}, \ldots, t_{n}\right):=V\left(t_{1}, \ldots, t_{\ell-1}, t_{\ell+1}, \ldots, t_{n}\right) \\
=\sum_{k=1}^{n-1} \sum_{\substack{j=1 \\
j \neq \ell}}^{n} \log \left|\left(t_{j}-\xi_{k}\right)\left(t_{j}-\bar{\xi}_{k}\right)\right|-2 \sum_{\substack{1 \leq j<k \leq n \\
j \neq \ell, k \neq \ell}} \log \left|t_{j}-t_{k}\right| .
\end{aligned}
$$

In Figure 2, particular sets of electrons and protons are shown along with the transformed configuration on the real axis. Namely, the zeros of the monic Blaschke product $B($.$) are 1 / 2,(1+i) / 2,2 / 3 i,-3 / 4 i$ and $-7 / 10+6 / 10 i$. The protons are at the critical points of this monic Blaschke product $B^{\prime}()=0:. 0.38-2.21 i, 1.69+1.13 i$, $0.68+1.86 i,-0.99+0.94 i,-0.53+0.51 i, 0.17+0.47 i, 0.41+0.27 i, 0.08-0.44 i$ (here and in the remaining part of this paragraph the numbers are rounded to two decimal digits). The electrons are at the solutions of $B()=$.1 , and their arguments are: $-2.87,-1.19,0.41,1.28,2.33$. For the inverse Cayley transform, $\theta=-2.87$, that is, the first electron is mapped to infinity.

In the next proposition we point out, how the critical points of the original and the transformed energy function correspond to each other.

Proposition 3.3. Let $\zeta_{j} \in \mathbb{D}, j=1, \ldots, n-1$ and $w_{j} \in \mathbb{C}, j=1, \ldots, n$. Assume that $w_{j}$ 's are restricted to the unit circle, i.e. (2.15) and (2.16) hold. We also assume that $\left(w_{1}, \ldots, w_{n}, \zeta_{1}, \ldots, \zeta_{n-1}\right) \notin E$. 



FIGURE 2. Equilibrium configurations of five electrons on the unit circle and the transformed configuration, with one electron transferred to $\infty$.

Fix $w_{1}$ and $\tau_{1} \in \mathbb{R}$ and assume that $\left(\tau_{1}, \tau_{2}, \ldots, \tau_{n}\right) \in A$. Consider the inverse Cayley mapping $C_{\tau_{1}}($.$) and also the points \xi_{j}:=C_{\tau_{1}}\left(\zeta_{j}\right), \overline{\xi_{j}}=C_{\tau_{1}}\left(\zeta_{j}^{*}\right)$ and $t_{j}=$ $C_{\tau_{1}}\left(e^{i \tau_{j}}\right)$.

Then $\tau_{2}<\ldots<\tau_{n}$ from the interval $\left(\tau_{1}, \tau_{1}+2 \pi\right)$ is a (real) critical point of $\widetilde{W}$ if and only if $t_{2}<\ldots<t_{n}$ is a (real) critical point of $V=V\left(t_{2}, \ldots, t_{n}\right)$.

Proof. Basically, we use the chain rule to show that the critical points correspond to each other under the diffeomorphism given by the inverse Cayley transform.

Let $\psi(\tau):=e^{i \tau}$. It is standard to see

$$
C_{\theta}(\psi(\tau))=i \frac{1+e^{i(\tau-\theta)}}{1-e^{i(\tau-\theta)}}=-\cot \frac{\tau-\theta}{2}, \quad \frac{d}{d \tau} C_{\theta}(\psi(\tau))=\frac{1}{\sin ^{2} \frac{\tau-\theta}{2}}
$$

where we used real differentiation with respect to $\tau$. We write $\Psi\left(\tau_{2}, \ldots, \tau_{n}\right):=$ $\left(\psi\left(\tau_{2}\right), \ldots, \psi\left(\tau_{n}\right)\right)$ and $K\left(z_{2}, \ldots, z_{n}\right):=\left(C_{\theta}\left(z_{2}\right), \ldots, C_{\theta}\left(z_{n}\right)\right)^{T}$, where $\cdot^{T}$ denotes transpose. Hence $K \circ \Psi$ maps from $\mathbb{R}^{n-1}$ to $\mathbb{R}^{n-1}$ and $\widetilde{W}=W \circ \Psi=V \circ K \circ \Psi+c$, by Proposition 2.3. The derivative of $K \circ \Psi$ as a real mapping is the diagonal matrix $D:=\operatorname{diag}\left(\sin ^{-2}\left(\frac{\tau_{2}-\theta}{2}\right), \ldots, \sin ^{-2}\left(\frac{\tau_{n}-\theta}{2}\right)\right)$. This is an invertible matrix, because $\theta=\tau_{1}<\tau_{2}<\ldots<\tau_{n}<\tau_{1}+2 \pi$. Because of chain rule,

$$
\nabla_{\tau_{2}, \ldots, \tau_{n}} \widetilde{W}=\left.\nabla_{t_{2}, \ldots, t_{n}} V\right|_{K \circ \Psi} \cdot D
$$

or by coordinates

$$
\frac{\partial \widetilde{W}\left(\tau_{2}, \ldots, \tau_{n}\right)}{\partial \tau_{j}}=\left.\frac{\partial V\left(t_{2}, \ldots, t_{n}\right)}{\partial t_{j}}\right|_{K \circ \Psi} \cdot \frac{1}{\sin ^{2}\left(\frac{\tau_{j}-\theta}{2}\right)}, \quad j=2, \ldots, n,
$$

which immediately implies the assertion.

\section{Proofs of the two main theorems.}

Proof of Theorem 1.1. We have that $\tau_{j}$ 's are different, and $a_{1}, \ldots, a_{n} \in \mathbb{D}$ is a sequence with $\zeta_{j} \neq 0$. These imply that $\left(\exp \left(i \tau_{1}(\delta)\right), \ldots, \exp \left(i \tau_{n}(\delta)\right), \zeta_{1}, \ldots, \zeta_{n-1}\right)$ is not in $E$ (see (2.1)). We also use the parametrization of the solution curve $S$ defined in (1.7), and the strict monotonicity and continuity of $\delta \mapsto \tau_{1}(\delta)$. Hence for any $w_{1}$, 
$w_{1}=e^{i \beta}$ where $\beta \in[0,2 \pi)$, the respective points on the solution curve $S$ are uniquely determined: $w_{j}=w_{j}\left(w_{1}\right)$, more precisely, $w_{j}=\exp \left(i \tau_{j}\left(\tau_{1}^{-1}(\beta)\right)\right), j=2, \ldots, n$.

Fix $w_{1}$, or, equivalently, $\beta \in[0,2 \pi)$. Now we want to show that

$$
\left(\tau_{2}, \tau_{3}, \ldots, \tau_{n}\right) \mapsto \widetilde{W}\left(\beta, \tau_{2}, \tau_{3}, \ldots, \tau_{n}\right)
$$

(assuming $\beta<\tau_{2}<\ldots<\tau_{n}<\beta+2 \pi$ ) has only one critical point, namely the point with $\tau_{j}=\tau_{j}\left(\tau_{1}^{-1}(\beta)\right)$ for $j=2,3, \ldots, n$, which happens to be the unique minimum point in $\left(\tau_{2}, \tau_{3}, \ldots, \tau_{n}\right)$.

To this end, we are going to transform the question to the upper half-plane, as we want to use Lemma 6 from [16]. We apply first the inverse Cayley transform $C()=.C_{\beta}($.$) which maps w_{1}$ to $\infty$. Hence we have $n-1$ pairs of fixed protons, $\xi_{j}=C\left(\zeta_{j}\right), \overline{\xi_{j}}=C\left(\zeta_{j}^{*}\right), j=1, \ldots, n-1$ and $n-1$ free electrons on the real axis, $t_{j}=C\left(e^{i \tau_{j}}\right), j=2, \ldots, n$. We know that $\beta<\tau_{2}<\ldots<\tau_{n}<\beta+2 \pi$, and $t_{2}<t_{3}<\ldots<t_{n}$ are equivalent. (If any two of the $\tau$ 's were equal, then the corresponding $t$ 's would be equal too and $\widetilde{W}\left(\tau_{2}, \ldots, \tau_{n}\right)=V\left(t_{2}, \ldots, t_{n}\right)=+\infty$, but we assumed that $\left(w_{1}, \ldots, w_{n}, \zeta_{1}, \ldots, \zeta_{n-1}\right) \notin E$ so that all $w_{j}$ 's have to be different.) Again, since we are outside $E$, we know that $\xi_{j} \neq-i$ and $\bar{\xi}_{j} \neq-i$, which, in turn, implies that $c$ is finite in (3.2). Thus, we can apply Proposition $3.2($ for $\ell=1)$ to relate the energy $\widetilde{W}$ on the unit circle and the energy $V$ on the real axis:

$$
\widetilde{W}\left(\beta, \tau_{2}, \ldots, \tau_{n}\right)=W\left(e^{i \beta}, e^{i \tau_{2}}, \ldots, e^{i \tau_{n}}\right)=V\left(t_{2}, \ldots, t_{n}\right)+c .
$$

Introducing $U:=\left\{\left(t_{2}, \ldots, t_{n}\right) \in \mathbb{R}^{n-1}: t_{2}<t_{3}<\ldots<t_{n}\right\}$, Lemma 6 from [16] gives that there is exactly one critical point $\left(\widetilde{t}_{2}, \ldots, \widetilde{t}_{n}\right)$ of $V$ in $U$ (gradient of $V$ vanishes), which is the global minimum point in $U$. In view of Proposition 3.3, the corresponding $\left(\widetilde{\tau_{2}}, \ldots, \widetilde{\tau_{n}}\right)$ with $\beta<\widetilde{\tau_{2}}<\ldots<\widetilde{\tau_{n}}<\beta+2 \pi$ and $\exp \left(i \widetilde{\tau_{2}}\right)=$ $C_{\beta}^{-1}\left(\widetilde{t_{2}}\right), \ldots, \exp \left(i \widetilde{\tau_{n}}\right)=C_{\beta}^{-1}\left(\widetilde{t_{n}}\right)$, is the only critical point of $\widetilde{W}=\widetilde{W}\left(\beta, \tau_{2}, \ldots, \tau_{n}\right)$, restricted to the simplex $\Delta_{\beta}$ of points of the form $\left(\beta, \tau_{2}, \ldots, \tau_{n}\right)$ under the condition $\beta<\tau_{2}<\tau_{3}<\ldots<\tau_{n}<\beta+2 \pi$. Note that $\Delta_{\beta}=Z_{\beta} \cap A$ with $Z_{\beta}$ denoting the hyperplane $\{\beta\} \times \mathbb{R}^{n-1}$. Furthermore, applying Proposition 3.2, we get that this is the unique global minimum point of $\widetilde{W}$ on $\Delta_{\beta}$.

Let us define $\varphi:[0,2 \pi) \rightarrow \mathbb{R}^{n}$ by putting $\varphi(\beta):=\left(\beta, \widetilde{\tau_{2}}, \widetilde{\tau_{3}}, \ldots, \widetilde{\tau_{n}}\right)$.

As $S$ is a continuous curve lying in $A$, there exists a point $\mathbf{t}$ of $S \cap Z_{\beta}$, which necessarily belongs to $S \cap Z_{\beta} \cap A=S \cap \Delta_{\beta}$, too. However - as it was shown in Theorem 4 in [15] $-\nabla \widetilde{W} \equiv \mathbf{0}$ on $S$, therefore $\mathbf{t}$ is also a critical point of $\left.\widetilde{W}\right|_{\Delta_{\beta}}$. Whence $\mathbf{t}=\varphi(\beta)$, the unique critical point of $\left.\widetilde{W}\right|_{\Delta_{\beta}}$, which is, as said above, the global minimum point of $\left.\widetilde{W}\right|_{\Delta_{\beta}}$, too.

It is easy to see that $\Phi:=W \circ \varphi$ is continuous on $[0,2 \pi)$ and with $\Phi(2 \pi):=$ $W(\varphi(0))$ is continuously extensible onto $[0,2 \pi]$. Thus $\Phi=W \circ \varphi$ has a global minimum on $[0,2 \pi)$, let it be $\beta^{*}$. Obviously, $\varphi\left(\beta^{*}\right)$ is also on the solution curve $S$, and $\widetilde{W}\left(\tau_{1}, \ldots, \tau_{n}\right)$ has a global minimum in $\varphi\left(\beta^{*}\right)$. Since $S$ is a smooth arc, and $\nabla \widetilde{W} \equiv \mathbf{0}$ on $S$, we get that $\left.\widetilde{W}\right|_{S} \equiv$ const. That is, we find $\left.\widetilde{W}\right|_{S} \equiv \varphi\left(\beta^{*}\right)$, the global minimum of the discrete energy function $\widetilde{W}=\widetilde{W}\left(\tau_{1}, \ldots, \tau_{n}\right)$.

Finally, we show that all points of $S_{\mathbb{R}}$ are global minimum points of $\widetilde{W}($.$) . Us-$ ing that $\widetilde{W}($.$) is (2 \pi, \ldots, 2 \pi)$-periodic, that is $\widetilde{W}\left(\tau_{1}, \tau_{2}, \ldots, \tau_{n}\right)=\widetilde{W}\left(\tau_{1}+2 \pi, \tau_{2}+\right.$ $\left.2 \pi, \ldots, \tau_{n}+2 \pi\right)$ and that for each $j, \tau_{j}(\delta+2 n \pi)=\tau_{j}(\delta)+2 \pi$, we obtain that $\widetilde{W}\left(\tau_{1}(\delta), \ldots, \tau_{n}(\delta)\right)$ is actually $2 n \pi$ periodic in $\delta$. This, expressed with $S$ and $S_{\mathbb{R}}$, implies that all points of $S_{\mathbb{R}}$ are global minimum points of $\widetilde{W}($.$) .$ 
Note that the above provides a positive answer to the question raised in [15], p. 476: the discrete energy function $\widetilde{W}=\widetilde{W}\left(\tau_{1}, \ldots, \tau_{n}\right)$ attains global minimum at every point of the full solution curve $S_{\mathbb{R}}$. Moreover, these are the only critical points of $\widetilde{W}$.

We collect the following set of "bad" configurations:

$$
X:=\left\{\left(z_{1}, z_{2}, \ldots, z_{n}\right) \in(\partial \mathbb{D})^{n}: z_{j}=z_{k} \text { for some } j \neq k \text {, or } B^{\prime}(0)=0\right\} .
$$

Proof of Theorem 1.2. Let $\left(z_{1}, \ldots, z_{n}\right) \in(\partial \mathbb{D})^{n} \backslash X$ be given. Denote their arguments by $t_{j}:=\Im \log \left(z_{j}\right), j=1,2, \ldots, n$. Without loss of generality, we may assume that $t_{1}, t_{2}, \ldots, t_{n} \in[0,2 \pi)$ and $t_{1}<t_{2}<\ldots<t_{n}$.

We use the above cited result of Hjelle providing a Blaschke product $B(z)=$ $B\left(z_{1}, \ldots, z_{n} ; z\right)$ with degree $n$, satisfying (1.9). Denote the leading coefficient of $B($. by $\chi$ where $\chi=e^{i \delta_{0}}$; note that $\delta_{0}$ is determined only $\bmod 2 \pi$ by this choice. Let us define $B_{1}(z):=\chi^{-1} B(z)$ which is the monic Blaschke product with the same zeros. We use $\alpha, T, S_{0}, S$ and $S_{\mathbb{R}}$ defined for $B_{1}($.$) . Now we fix the value of \delta_{0}$ so that $-\delta_{0} \in[\alpha, \alpha+2 \pi)$; observe that this does not change the value of $\chi$ and does not cause circular dependence. Note that the sets $S_{\mathbb{R}}$ defined for $B$ and $B_{1}$ are the same, because multiplying the Blaschke product with a constant is just a translation of variable. More precisely $\tau_{j}(B ; \delta)=\tau_{j}\left(B_{1} ; \delta-\delta_{0}\right)$ for all $j=1,2, \ldots, n, \delta \in \mathbb{R}$.

Hjelle's result means that $\tau_{j}(B ; 0)=t_{j}$, hence $\tau_{j}\left(B_{1} ;-\delta_{0}\right)=t_{j}$. By the choice of $\delta_{0}$, we immediately see that $\left(t_{1}, t_{2}, \ldots, t_{n}\right)=T\left(-\delta_{0}\right)$, that is, $\left(t_{1}, t_{2}, \ldots, t_{n}\right)$ is on $S_{0}$ defined in (1.6) for the monic Blaschke product $B_{1}($.$) .$

We use the description from Theorem 1.1. This way we obtain that $\widetilde{W}($.$) has$ global minimum at the points $T(\delta), \delta \in\left[\alpha, \alpha+2 \pi\right.$ ) (defined by $B_{1}($.$) ). Observe$ that when the parameter $\delta$ changes continuously further on in $[\alpha, \alpha+2 n \pi)$, the curve $T(\delta)$ recovers $(\bmod 2 \pi)$ the same set of arguments $\left(t_{1}, \ldots, t_{n}\right) n$ times, in each cyclic permutations of them, while the corresponding $z_{1}, \ldots, z_{n}$ is repeated $n$ times (in each cyclic order of the values) always determining the same Blaschke product.

We remark, that according to Proposition 2.5, the energy function $W($.$) has$ critical point in $\left(z_{1}, z_{2}, \ldots, z_{n}\right)$ not just with restriction to the unit circle, but also in the total electrostatic equilibrium sense. This was also observed in [14], see Theorem 2 .

Roughly speaking, the union of solution curves for different $a_{1}, a_{2}, \ldots, a_{n}$ covers the whole $A \cap Q$, and considering as electrons on the unit circle, the whole space $\left(z_{1}, z_{2}, \ldots, z_{n}\right) \in(\partial \mathbb{D})^{n} \backslash X$.

This last result, when compared with Theorem 1.1, shows a direct relation between the location of electrons, $z_{1}, z_{2}, \ldots, z_{n}$ and the location of pairs of protons, $\zeta_{1}, \zeta_{1}^{*}, \zeta_{2}, \zeta_{2}^{*}, \ldots, \zeta_{n-1}, \zeta_{n-1}^{*}$.

Corollary 4.1. If $\left(z_{1}, \ldots, z_{n}\right) \in(\partial \mathbb{D})^{n} \backslash X$ is given, then the points $\zeta_{1}, \ldots, \zeta_{n-1} \in$ $\mathbb{D} \backslash\{0\}$ in Theorem 1.2 are the critical points of the Blaschke product satisfying (1.9).

Acknowledgments. The authors gratefully acknowledge their indebtedness to Margit Pap and Ferenc Schipp for calling their attention to the problem and for useful suggestions and discussions.

This research was partially supported by the DAAD-TKA Research Project "Harmonic Analysis and Extremal Problems" \# 308015.

Marcell Gaál was supported by the National, Research and Innovation Office NKFIH Reg. No.'s K-115383 and K-128972, and also by the Ministry for Innovation 
and Technology, Hungary throughout Grant TUDFO/47138-1/2019-ITM.

Béla Nagy was supported by the ÚNKP-18-4 New National Excellence Program of the Ministry of Human Capacities.

Zsuzsanna Nagy-Csiha was supported by the ÚNKP-19-3 New National Excellence Program of the Ministry for Innovation and Technology.

Szilárd Gy. Révész was supported in part by Hungarian National Research, Development and Innovation Fund projects \# K-119528 and K-132097.

The authors are grateful to the anonymous referees for their thorough work, precise corrections and useful suggestions.

The authors are thankful to Gunter Semmler for his interest and constructive remarks.

\section{REFERENCES}

[1] A. Bultheel, P. GonzÁlez-Vera, E. Hendriksen, and O. NjÅstad, Orthogonal rational functions, vol. 5 of Cambridge Monographs on Applied and Computational Mathematics, Cambridge University Press, Cambridge, 1999, https://doi.org/10.1017/ CBO9780511530050, https://doi.org/10.1017/CBO9780511530050.

[2] D. G. Cantor and R. R. Phelps, An elementary interpolation theorem, Proc. Amer. Math. Soc., 16 (1965), pp. 523-525, https://doi.org/10.2307/2034689.

[3] H. G. Feichtinger AND M. PAP, Hyperbolic wavelets and multiresolution in the Hardy space of the upper half plane, in Blaschke products and their applications, vol. 65 of Fields Inst. Commun., Springer, New York, 2013, pp. 193-208, https://doi.org/10.1007/ 978-1-4614-5341-3_11.

[4] P. J. Forrester And J. B. Rogers, Electrostatics and the zeros of the classical polynomials, SIAM J. Math. Anal., 17 (1986), pp. 461-468, https://doi.org/10.1137/0517035.

[5] L. GolinskiI, Quadrature formula and zeros of para-orthogonal polynomials on the unit circle, Acta Math. Hungar., 96 (2002), pp. 169-186, https://doi.org/10.1023/A:1019765002077, https://doi.org/10.1023/A:1019765002077.

[6] F. A. GrünBAum, Variations on a theme of Heine and Stieltjes: an electrostatic interpretation of the zeros of certain polynomials, in Proceedings of the VIIIth Symposium on Orthogonal Polynomials and Their Applications (Seville, 1997), vol. 99, 1998, pp. 189-194, https: //doi.org/10.1016/S0377-0427(98)00156-3.

[7] P. S. C. Heuberger, P. M. J. Van Den Hof, and B. Wahlberg, eds., Modelling and identification with rational orthogonal basis functions, Springer-Verlag London, Ltd., London, 2005, https://doi.org/10.1007/1-84628-178-4.

[8] G. A. HJElle, Constructing interpolating Blaschke products with given preimages, Comput. Methods Funct. Theory, 7 (2007), pp. 43-54, https://doi.org/10.1007/BF03321630.

[9] M. E. H. Ismail, An electrostatics model for zeros of general orthogonal polynomials, Pacific J. Math., 193 (2000), pp. 355-369, https://doi.org/10.2140/pjm.2000.193.355, https://doi. org/10.2140/pjm.2000.193.355.

[10] M. E. H. IsmaIL, More on electrostatic models for zeros of orthogonal polynomials, in Proceedings of the International Conference on Fourier Analysis and Applications (Kuwait, 1998), vol. 21, 2000, pp. 191-204, https://doi.org/10.1080/01630560008816948, https: //doi.org/10.1080/01630560008816948.

[11] W. B. Jones And S. RuscheWeyh, Blaschke product interpolation and its application to the design of digital filters, Constr. Approx., 3 (1987), pp. 405-409, https://doi.org/10.1007/ BF01890578.

[12] A. Martinez-Finkelshtein, P. Martinez-González, and R. Orive, Asymptotics of polynomial solutions of a class of generalized Lamé differential equations, Electron. Trans. Numer. Anal., 19 (2005), pp. 18-28.

[13] W. Mi, T. QIAN, AND F. WAN, A fast adaptive model reduction method based on TakenakaMalmquist systems, Systems Control Lett., 61 (2012), pp. 223-230, https://doi.org/10. 1016/j.sysconle.2011.10.016.

[14] M. PAP AND F. SChIPP, Malmquist-Takenaka systems and equilibrium conditions, Math. Pannon., 12 (2001), pp. 185-194.

[15] M. PAP AND F. SCHIPP, Equilibrium conditions for the Malmquist-Takenaka systems, Acta Sci. Math. (Szeged), 81 (2015), pp. 469-482, https://doi.org/10.14232/actasm-015-765-6.

[16] G. Semmler And E. Wegert, Finite Blaschke products with prescribed critical points, Stieltjes 
polynomials, and moment problems, Anal. Math. Phys., 9 (2019), pp. 221-249, https: //doi.org/10.1007/s13324-017-0193-5.

[17] T. Sheil-Small, Complex polynomials, vol. 75 of Cambridge Studies in Advanced Mathematics, Cambridge University Press, Cambridge, 2002, https://doi.org/10.1017/ CBO9780511543074.

[18] B. SimAneK, An electrostatic interpretation of the zeros of paraorthogonal polynomials on the unit circle, SIAM J. Math. Anal., 48 (2016), pp. 2250-2268, https://doi.org/10.1137/ 151005415 . 